\title{
Retraction of: Optimization of Planning and Design of Urban Sewage Collection and Treatment Systems, by Tian J, Cheng J, and Gong Y. Environ Eng Sci. 2018;35(7):760-769; DOI: $10.1089 /$ ees.2017.0019
}

$\mathbf{T}$

He Published article entitled, Optimization of Planning and Design of Urban Sewage Collection and Treatment Systems, by J. Tian, J. Cheng, and Y. Gong. Environ Eng Sci. 35(7):760-769; DOI: 10.1089/ees.2017.0019 is being officially retracted from Environmental Engineering Science due to the discovery by the authors of significant errors with the programming used in the paper.

After the publication of the article, when applying the same program used in the published paper to optimize urban sewage collection and treatment in city in China, the authors found faults within the program. They decided to recalculate examples in the published paper and the new results were significantly different from those that had been reported in the article. Consequently, the authors stated that the "paper is no longer suitable for publication," and requested that it be retracted, to which the Editor of Environmental Engineering Science agreed.

Environmental Engineering Science is committed to upholding the highest standards of scientific publishing and commend the authors for bringing this to the Editor's attention. 